\title{
Heterogeneity Matters When Modeling COVID-19
}

\author{
A new model shows that restricting the number of social interactions \\ among members of a population is effective at controlling outbreaks \\ dominated by "superspreaders," explaining the unexpected success of \\ last year's lockdowns.
}

By Katherine Wright

lo n early 2020, many countries implemented

lockdowns to help curb the spread of COVID-19. At the time, models suggested that these lockdowns would have only a moderate effect on infection numbers. But the strategy proved surprisingly effective, says Kim Sneppen, a physicist who studies epidemics at Copenhagen University in Denmark. Wanting to understand the reasons for this unexpected outcome, Sneppen and his colleagues looked at their models again. Their results, published today, show that the discrepancy can be explained by infection-rate heterogeneity-something that wasn't considered a year ago but that's now known to be a characteristic of COVID-19 [1]. Factoring in this heterogeneity leads to a sharp increase in the predicted effectiveness of mitigation strategies that limit a population's social interactions.

The spread of flu is fairly easy to capture with a standard

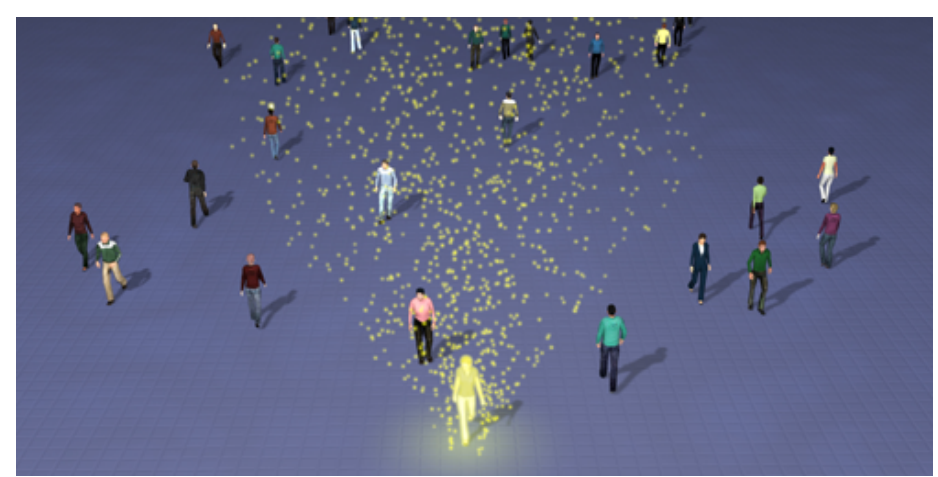

Credit: vrx123/stock.adobe.com epidemic model because its infection rate-the number of people who catch the illness from each infected individual-varies little from person to person. Initially, epidemiologists thought that the infection rate of COVID-19, while higher than that of flu, was equally uniform. But as 2020 progressed, it became apparent that wasn't the case. Instead, some COVID-19 carriers infect no one, while others, known as superspreaders, infect whole crowds.

The team included such a variable infection rate in an agent-based COVID-19 model that treats the population as a collection of autonomous decision-making entities. They then used the model to test the efficacy of various mitigation strategies. They found that limiting the number of social interactions between groups of people-something that happens during lockdowns-rather than their duration was the best way to halt the disease's spread. The same mitigation strategy would not work for flu.

Katherine Wright is a Senior Editor for Physics.

\section{REFERENCES}

1. B. F. Nielsen et al., "COVID-19 superspreading suggests mitigation by social network modulation," Phys. Rev. Lett. 126, 118301 (2021). 